\title{
Direct Methods for Linear Systems with Inexact Input Data
}

\section{Günter MAYER}

Institut für Mathematik, Universität Rostock

Universitätsplatz 1, D-18051 Rostock, Germany

E-mail: guenter.mayer@uni-rostock.de

Received February 15, 2008

Revised October 31, 2008

\begin{abstract}
We give a survey on direct methods for interval linear systems. We also consider various kinds of solution sets and show how the interval hull can be computed.
\end{abstract}

Key words: interval linear system, solution set, interval hull, interval Gaussian algorithm, interval Cholesky method, interval Trench algorithm, interval Bareiss algorithm

\section{Introduction}

We consider systems

$$
A x=b
$$

of linear equations where $A$ is a real $n \times n$ matrix and $b$ is a real vector with $n$ components. Both are allowed to vary within bounds $\underline{A}, \bar{A}, \underline{b}, \bar{b}$, respectively, which form an interval matrix $[A]=[\underline{A}, \bar{A}]$ and an interval vector $[b]=[\underline{b}, \bar{b}]$. One writes

$$
[A] x=[b]
$$

for the set of such systems and speaks of interval linear systems [50] or of linear interval systems [41], where linearity only refers to the single systems (1.1) contained in (1.2) and not to an algebraic interpretation of (1.2). In fact, $[A] \cdot x$ is generally not a linear mapping even when restricting $x$ to real point vectors. Leontief's static open input-output model with varying input coefficients reduces to (1.2), e.g., and a Taylor expansion of nonlinear functions $f: \mathbb{R}^{n} \rightarrow \mathbb{R}^{n}$ at a given approximation $\tilde{x}$ of a zero $x^{*}$ of $f$ also results in it, when the intermediate points $\xi_{i}$ should be enclosed by given bounds; cf. [1].

Defining the solution set

$$
\Sigma=\{x \mid(\exists A \in[A])(\exists b \in[b])(A x=b)\},
$$

we are interested in interval methods which produce an output vector $[x]$ with $\Sigma \subseteq[x]$. Sometimes only systems with matrices in $[A]$ of a certain structure are of interest. Then we look for interval methods with an output vector $[x]^{\text {struc }}$ containing the corresponding structured solution set

$$
\begin{aligned}
\Sigma^{\text {struc }} & =\{x \mid(\exists A \in[A])(\exists b \in[b])(A x=b, A \text { structured in a prescribed way })\} \\
& \subseteq \Sigma .
\end{aligned}
$$


In particular, we will consider the symmetric solution set

$$
\Sigma^{\mathrm{sym}}=\left\{x \mid(\exists A \in[A])(\exists b \in[b])\left(A x=b, A=A^{\mathrm{T}}\right)\right\},
$$

where $[A]$ is assumed to satisfy $[A]=[A]^{\mathrm{T}}$, and the Toeplitz solution set

$$
\Sigma^{\text {Toep }}=\{x \mid(\exists A \in[A])(\exists b \in[b])(A x=b, A \text { Toeplitz matrix })\},
$$

where similarly $[a]_{i j}$ is assumed to be constant for $|i-j|=$ const.

In this paper we briefly characterize selected solution sets. We also cite results for the complexity of computing the interval hull of $\Sigma$. Moreover, we consider direct interval methods for enclosing $\Sigma, \Sigma^{\mathrm{sym}}$ and $\Sigma^{\text {Toep }}$ by interval vectors. In particular, we give a survey of theoretical results for the interval Gaussian algorithm, the interval Cholesky method and interval algorithms for systems with Toeplitz matrices. We also investigate connections between the first two algorithms. In the whole paper we tacitly assume that $[A]$ is regular, i.e., each system (1.1) with $A \in[A]$ is uniquely solvable.

We have organized our paper as follows: In Section 2 we present our notation, in Section 3 we study selected solution sets, in Section 4 we consider the interval hull and results on complexity. Section 5 is devoted to the interval Gaussian algorithm while Section 6 refers to the interval Cholesky method. In Section 7 we modify Garloff's results on interval Toeplitz matrices and in Section 8 we list some additional expressions which enclose $\Sigma$.

\section{Notation}

By $\mathbb{R}^{n}, \mathbb{R}^{n \times n}, \mathbb{I} \mathbb{R}, \mathbb{I}^{n}, \mathbb{I}^{n \times n}$ we denote the set of real vectors with $n$ components, the set of real $n \times n$ matrices, the set of intervals, the set of interval vectors with $n$ components and the set of $n \times n$ interval matrices, respectively. By "interval" we always mean a real compact interval. We write interval quantities in brackets with the exception of point quantities (degenerate interval quantities) which we identify with the element which they contain. Examples are the vector $e=(1,1, \ldots, 1)^{\mathrm{T}}$, the zero matrix $O$, the identity matrix $I$ with its $k$-th column $e^{(k)}$, and the matrix $E$ which has ones along the counter-diagonal and zeros otherwise. We use the notation $[A]=[\underline{A}, \bar{A}]=\left([a]_{i j}\right)=\left(\left[\underline{a}_{i j}, \bar{a}_{i j}\right]\right) \in \mathbb{I}^{n \times n}$ simultaneously without further reference, and we proceed similarly for the elements of $\mathbb{R}^{n}, \mathbb{R}^{n \times n}$, $\mathbb{I} \mathbb{R}$ and $\mathbb{I}^{n}$. For an interval $[a]$ we write $\check{a}$ for the midpoint, $\operatorname{rad}([a])$ for the radius, $|[a]|$ for the absolute value, and $\langle[a]\rangle$ for the minimal absolute value. For $n \times n$ interval matrices $[A]$ we apply these operators entrywise with the exception of the last one. Here, the comparison matrix (Ostrowski matrix) $\langle[A]\rangle=\left(c_{i j}\right)$ is defined by $c_{i j}=-\left|[a]_{i j}\right|$ if $i \neq j$ and $c_{i j}=\left\langle[a]_{i i}\right\rangle$ if $i=j$. For interval vectors we proceed similarly. Since real numbers can be viewed as degenerate intervals, $|\cdot|$ and $\langle\cdot\rangle$ can also be used for them. So a diagonal matrix $D \in \mathbb{R}^{n \times n}$ is a signature matrix if $|D|=I$. If $S \subseteq \mathbb{R}^{n}$ is an arbitrary bounded set the tightest interval vector $\square S$ which encloses $S$ is called interval hull of $S$. 
By $A \geq O$ we denote a non-negative $n \times n$ matrix, i.e., $a_{i j} \geq 0$ for $i, j=1, \ldots, n$. For vectors $x \in \mathbb{R}^{n}$ we proceed analogously. We call $x$ positive writing $x>0$ if $x_{i}>0$, $i=1, \ldots, n$. We use $Z^{n \times n}$ for the set of real $n \times n$ matrices with non-positive offdiagonal entries. As usual we call $A \in \mathbb{R}^{n \times n}$ an $M$-matrix if $A$ is non-singular with $A^{-1} \geq O$ and $A \in Z^{n \times n}$. It is an $H$-matrix if $\langle A\rangle$ is an $M$-matrix. By $\rho(A)$ we denote the spectral radius of $A$ and for a given vector $v \in \mathbb{R}^{n}$ we write $\overleftarrow{v}$ for the vector $E v=\left(v_{n}, \ldots, v_{1}\right)^{\mathrm{T}}$.

An interval matrix $[A] \in \mathbb{I} \mathbb{R}^{n \times n}$ is an $M$-matrix if each element $\tilde{A} \in[A]$ is an $M$-matrix. In the same way the terminology " $H$-matrix" can be extended to $\mathbb{R}^{n \times n}$. It is easy to verify that $[A]$ is an $H$-matrix if and only if $\langle[A]\rangle$ is an $M$-matrix.

We call $[A] \in \mathbb{I}^{n \times n}, n>1$, irreducible if $\langle[A]\rangle$ is irreducible. If there is a positive vector $x$ such that $\langle[A]\rangle x \geq 0$ holds then we call $[A]$ generalized diagonally dominant. Analogously we define generalized strictly diagonally dominant and generalized irreducibly diagonally dominant. If $x$ can be chosen to be $e$ then the specification "generalized" can be dropped. Cf. [25] for details. It is well-known that generalized strictly diagonally dominant matrices are $H$-matrices and vice versa.

We equip $\mathbb{R}, \mathbb{I} \mathbb{R}^{n}, \mathbb{I}^{n \times n}$ with the usual real interval arithmetic as described in [1], [32]. We assume that the reader is familiar with the basic properties of this arithmetic. For $[a] \in \mathbb{I} \mathbb{R}$ we recall the definitions $\sqrt{[a]}=[a]^{1 / 2}=\{\sqrt{a} \mid a \in[a]\}$ for $0 \leq \underline{a}$, and $[a]^{2}=\left\{a^{2} \mid a \in[a]\right\}$.

\section{Solution sets}

We first consider the general solution set $\Sigma$ as defined in (1.3). It can equivalently be characterized as

$$
x \in \Sigma \Longleftrightarrow[A] x \cap[b] \neq \emptyset \Longleftrightarrow|\check{A} x-\check{b}| \leq \operatorname{rad}([A]) \cdot|x|+\operatorname{rad}([b]),
$$

where the second characterization is due to Beeck [10] and the last one due to Oettli and Prager [36]. From the Oettli-Prager criterion one can see that in each fixed orthant $O$ the solution set $\Sigma$ is the intersection of finitely many half-spaces. In particular, $\Sigma \cap O$ is convex, compact, and connected [10], where the latter two properties also hold for $\Sigma$ as a whole. (For singular systems this may become false as Jansson's example $[-1,1] x=1$ shows immediately.) Thus $\Sigma$ is nonconvex in general, while its intersection with each orthant is either empty or a convex polyhedron.

The symmetric solution set $\Sigma^{\mathrm{sym}}$ behaves differently from $\Sigma$. Using a FourierMotzkin like process one can show that $\Sigma^{\text {sym }}$ is bounded curvilinearly. In fact its boundary is formed by pieces of algebraic hypersurfaces of order 1 and 2; cf. [2], [5]. The skew-symmetric solution set

$$
\Sigma^{\text {skew }}=\left\{x \mid(\exists A \in[A])(\exists b \in[b])\left(A x=b, A=-A^{\mathrm{T}}\right)\right\}
$$

with $[A]=-[A]^{\mathrm{T}}$ and zero diagonal, and the persymmetric solution set

$$
\Sigma^{\text {per }}=\left\{x \mid(\exists A \in[A])(\exists b \in[b])\left(A x=b, E A=(E A)^{\mathrm{T}}\right)\right\}
$$


with $E[A]=(E[A])^{\mathrm{T}}$ are bounded in the same way as $\Sigma^{\mathrm{sym}} ; \mathrm{cf}$. [2]. Things change for $\Sigma^{\text {Toep }}$, where again algebraic hypersurfaces are involved, but without a simple limitation of the order; see [3].

We now illustrate certain solution sets for the matrix $[A]=\left(\begin{array}{cc}1 & {[0,1]} \\ {[0,1]} & {[-4,-1]}\end{array}\right)$ and the vector $[b]=([0,2],[0,2])^{\mathrm{T}}$.

In Fig. 1 we also mentioned two solution sets which are not described up to now. While $\Sigma$ is defined in (1.3) using twice the existential qualifier there are also situations where one must apply the universal qualifier. In this way the tolerance solution set $\Sigma_{\forall \exists}=\{x \mid(\forall A \in[A])(\exists b \in[b])(A x=b)\}$ and the control solution set $\Sigma_{\exists \forall}=\{x \mid(\forall b \in[b])(\exists A \in[A])(A x=b)\}$ can be defined. Analogously to (3.1) one can prove the equivalences

$$
\begin{aligned}
& x \in \Sigma_{\forall \exists} \Longleftrightarrow[A] x \subseteq[b] \Longleftrightarrow|\check{b}-\check{A} x| \leq \operatorname{rad}([b])-\operatorname{rad}([A])|x| \quad([41]), \\
& x \in \Sigma_{\exists \forall} \Longleftrightarrow[A] x \supseteq[b] \Longleftrightarrow|\check{b}-\check{A} x| \leq \operatorname{rad}([A])|x|-\operatorname{rad}([b]) \quad([58]),
\end{aligned}
$$

which show that these sets are again bounded by pieces of hyperplanes. For applications see, e.g., [26], [46] and the literature therein.

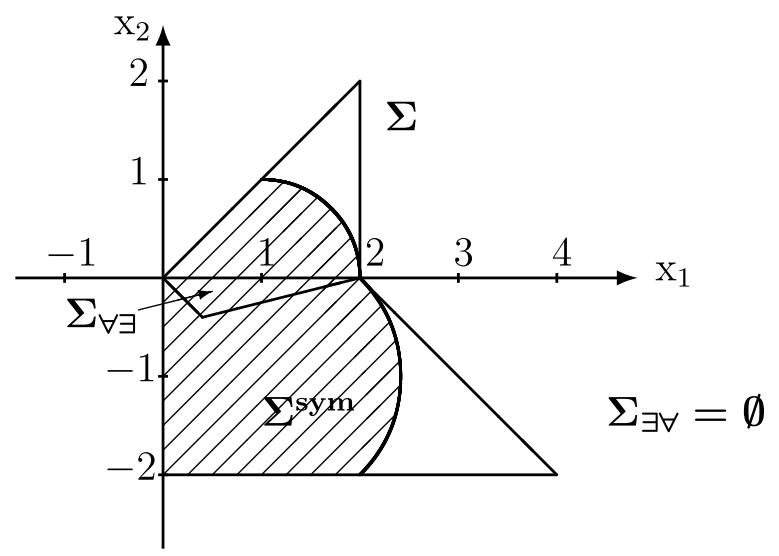

Fig. 1. Solution sets

\section{Complexity results and interval hull}

Verifying whether a given vector $x$ belongs to the solution set $\Sigma$ can be performed in polynomial time as can be seen from the Oettli-Prager criterion (3.1). Computing, however, the boundary of $\Sigma$ and also of $\Sigma^{\text {sym }}, \Sigma^{\text {skew }}, \Sigma^{\text {per }}$ is NP-hard [22], [46], [47].

In [46] it is mentioned that the problem of checking whether a system $[A] x=[b]$ has a tolerance solution can be performed in polynomial time while the problem of checking whether the control solution set $\Sigma_{\exists \forall}$ is non-empty is NP-complete.

By virtue of the somewhat irregular structure of $\Sigma$ combined with a difficult description one often contents oneself with interval bounds for $\Sigma$. In this section 
we will present results forming optimal interval bounds for $\Sigma$, i.e., its interval hull $\square \Sigma$. First we remark that the computation of this interval vector is NP-hard [46]. Nevertheless, for some particular classes of matrices $[A]$ we have success in polynomial time. We start with a generalization of the Hansen-Bliek-Rohn theorem ([12], [18], [33], [43]).

Theorem 4.1 ([46]). Let $[A]=\check{A}+[-R, R] \in \mathbb{R}^{n \times n}, \quad[b] \in \mathbb{I}^{n}, \quad R \geq O$, $\rho\left(\left|\check{A}^{-1}\right| R\right)<1, M=\left(I-\left|\check{A}^{-1}\right| R\right)^{-1}, x^{c}=\check{A}^{-1} \check{b}, x^{*}=M\left(\left|x_{c}\right|+\left|\check{A}^{-1} \operatorname{rad}([b])\right|\right)$. For each $i \in\{1, \ldots, n\}$ compute ${\underset{\sim}{i}}_{i}=-x_{i}^{*}+m_{i i}\left(x^{c}+\left|x^{c}\right|\right)_{i}, \tilde{x}_{i}=x_{i}^{*}+m_{i i}\left(x^{c}-\left|x^{c}\right|\right)_{i}$, $\nu_{i}=\frac{1}{2 m_{i i}-1} \in(0,1], \underline{x}_{i}=\min \left\{{\underset{\sim}{x}}_{i}, \nu_{i} x_{i}\right\}, \bar{x}_{i}=\max \left\{\tilde{x}_{i}, \nu_{i} \tilde{x}_{i}\right\}$. Then $\square \Sigma \subseteq[x]$ with equality if $\check{A}$ is a diagonal matrix with positive entries.

In [46] vectors $\underline{d}, \bar{d}$ are computed such that the enclosures $\underline{x} \leq \underline{x}^{H} \leq \underline{x}+\underline{d}$ and $\bar{x}-\bar{d} \leq \bar{x}^{H} \leq \bar{x}$ hold for $[x]^{H}=\square \Sigma$ and $[x]$ from Theorem 4.1. Moreover it is mentioned that the bounds $\underline{x}, \bar{x}$ in this theorem are at least as good as the componentwise Bauer-Skeel bounds (cf. [59]) and better in each entry provided that $\left(\left|\breve{A}^{-1}\right| R\right)_{i i}>0$ holds for each $i$.

Theorem 4.2 ([35]). Let $[A] \in \mathbb{R}^{n \times n}$ be an $H$-matrix, $[b] \in \mathbb{R}^{n}$, $u=$ $\langle[A]\rangle^{-1}|[b]|$. For each $i \in\{1, \ldots, n\}$ compute $d_{i}=\left(\langle[A]\rangle^{-1}\right)_{i i}, \alpha_{i}=\left\langle[a]_{i i}\right\rangle-1 / d_{i}$, $\beta_{i}=u_{i} / d_{i}-\left|[b]_{i}\right|$. Then

$$
(\square \Sigma)_{i} \subseteq[x]_{i}=\frac{[b]_{i}+\left[-\beta_{i}, \beta_{i}\right]}{[a]_{i i}+\left[-\alpha_{i}, \alpha_{i}\right]}, \quad i=1, \ldots, n,
$$

with $\square \Sigma=[x]$ if $\check{A}$ is diagonal.

Note that for $\check{A}=I$ (which occurs, e.g., when preconditioning (1.2) with $\check{A}^{-1}$ ) the Theorems 4.1 and 4.2 are equivalent in contrast to general midpoints $\breve{A}$ as the example $A=\check{A}=\left(\begin{array}{cc}2 & 1 \\ -1 & 2\end{array}\right), b=\check{b}=\left(\begin{array}{l}3 \\ 1\end{array}\right)$ shows.

TheOREM 4.3 ([4], [9], [11], [16]). Let $[A] \in \mathbb{I}^{n \times n}$ be an interval $M$-matrix and let $[b] \in \mathbb{I}^{n}$ satisfy $\underline{b} \geq 0$ or $0 \in[b]$ or $\bar{b} \leq 0$. Then each of the algorithms in the Sections 5-7 yields the interval hull $\square \Sigma$, where we assume $[A]=[A]^{\mathrm{T}}$ for the interval Cholesky method in Section 6 and $[a]_{i, i+k}=[\tau]_{k}, k=-n+1, \ldots, 0, \ldots, n-1$ for the interval Toeplitz methods in Section 7. In particular we get

$$
\square \Sigma= \begin{cases}{\left[\bar{A}^{-1} \underline{b}, \underline{A}^{-1} \bar{b}\right],} & \text { if } \underline{b} \geq 0, \\ {\left[\underline{A}^{-1} \underline{b}, \underline{A}^{-1} \bar{b}\right],} & \text { if } 0 \in[b], \\ {\left[\underline{A}^{-1} \underline{b}, \bar{A}-1 \bar{b}\right],} & \text { if } \bar{b} \leq 0 .\end{cases}
$$

Moreover, under the above-mentioned restrictions on $[A]$ we have $\square \Sigma=\square \Sigma^{\mathrm{sym}}$ and $\square \Sigma=\square \Sigma^{\text {Toep }}$, respectively.

The representation in (4.1) remains true if $[A]$ is weakened to be inverse positive, i.e., $A^{-1} \geq O$ for all $A \in[A]$ (without the restriction $A \in Z^{n \times n}$ ). 
Now we address Rohn's sign accord algorithm [32], [42] in order to find a solution $x^{D}$ of the nonlinear system

$$
\check{A} x-\check{b}=D(\operatorname{rad}([A])|x|+\operatorname{rad}([b])),
$$

where $D \in \mathbb{R}^{n \times n}$ is an arbitrarily given signature matrix. Taking absolute values on both sides one sees at once by virtue of the Oettli-Prager criterion (3.1) that each solution of (4.2) is in $\Sigma$. Moreover, knowing the solution of (4.2) for all signature matrices $D$ leads to $\square \Sigma$ by the following theorem.

TheOrem $4.4([42])$. Let $[A] \in \mathbb{I}^{n \times n},[b] \in \mathbb{R}^{n}$ and $X=\left\{x^{D}|| D \mid=I\right.$, $x^{D}$ solves (4.2)\}. Then the convex hulls of $X$ and $\Sigma$ coincide and $\underline{x}^{H}=\inf X$, $\bar{x}^{H}=\sup X$, where $[x]^{H}=\square \Sigma$ and where inf and sup are applied componentwise. At most $2^{2 n}$ linear systems have to be solved for computing $\square \Sigma$.

The complexity in Theorem 4.4 is essentially based on the following algorithm.

Algorithm 1. Rohn's sign accord algorithm [32], [42], [46]

Step 0. Select $D^{\prime} \in \mathbb{R}^{n \times n}$ with $\left|D^{\prime}\right|=I$. (Recommended: $D^{\prime}$ such that $D^{\prime}\left(\check{A}^{-1}(\check{b}+\right.$ $D \operatorname{rad}([b]))) \geq 0$.)

Step 1. Solve $\left(\check{A}-D \operatorname{rad}([A]) D^{\prime}\right) x=\check{b}+D \operatorname{rad}([b])$.

Step 2. If $D^{\prime} x \geq 0$ set $x^{D}:=x$ and terminate.

Step 3. Find $k=\min \left\{j \mid d_{j j}^{\prime} x_{j}<0\right\}$.

Step 4. Set $d_{k k}^{\prime}:=-d_{k k}^{\prime}$ and go to Step 1 .

Theorem $4.5([42])$. Let $[A] \in \mathbb{I}^{n \times n}$ and $[b] \in \mathbb{I}^{n}$. Then the sign accord algorithm is finite for each signature matrix $D$ and each starting signature matrix $D^{\prime}$. It terminates with a vector $x^{D}$ which satisfies (4.2). The vector $x^{D}$ does not depend on $D^{\prime}$ and is unique. There are at most $2^{n}$ linear systems to be solved.

This shows that the sign accord algorithm can be used for computing the hull of $\Sigma$. Alternatives are given in [21] and [57].

We mention that the number of linear systems in Theorems 4.4 and 4.5 is very pessimistic. In practice the amount of work is often much less - in particular, if one of the following properties holds: (i) $\Sigma$ is completely contained in the interior of a single orthant, (ii) $\operatorname{rad}([A])=u v^{\mathrm{T}}$ with nonnegative vectors $u, v$, (iii) $[A]$ is inverse sign stable, i.e., $\left|A^{-1}\right|>O$ for each $A \in[A]$. See [32], [46] for details.

\section{Interval Gaussian algorithm}

One of the well-known direct methods for solving (1.2) is the interval Gaussian algorithm. It produces an interval vector $[x]^{G}$ which contains $\Sigma$ and which is the topic of this section. The explicit formulae are, roughly speaking, the formulae of the conventional Gaussian algorithm, where the real entries and operations are replaced by intervals and corresponding interval operations. Since, in general, the 
solution set $\Sigma$ is not an interval vector, and also due to data dependency, $[x]^{G}$ often overestimates this set (cf. [30], [45], [54], [61]). But there are also classes of input data $[A],[b]$ such that $[x]^{G}$ results in the interval hull of $\Sigma$-at least if roundings are excluded; cf. for instance [9]. The conventional Gaussian algorithm without pivoting may break down by division by zero. If this failure does not occur we call the algorithm feasible, otherwise infeasible. This terminology is also used for the interval Gaussian algorithm where "division by zero" is replaced by "division by an interval which contains zero."

The algorithm starts with $[A]^{(1)}=[A] \in \mathbb{I}^{n \times n},[b]^{(1)}=[b] \in \mathbb{R}^{n}$ and-if it is feasible - results in a final vector $[x]^{G}=\operatorname{IGA}([A],[b]) \in \mathbb{R}^{n}$ via intermediate quantities $[A]^{(k)},[b]^{(k)}, k=2, \ldots, n$, in the following way:

$$
\begin{aligned}
& {[a]_{i j}^{(k+1)}=\left\{\begin{array}{ll}
{[a]_{i j}^{(k)},} & i=1, \ldots, k, j=1, \ldots, n, \\
{[a]_{i j}^{(k)}-\frac{[a]_{i k}^{(k)}[a]_{k j}^{(k)}}{[a]_{k k}^{(k)}},} & i, j=k+1, \ldots, n, \\
0 & \text { otherwise, }
\end{array}\right\} k=1, \ldots, n-1,} \\
& {[b]_{i}^{(k+1)}=\left\{\begin{array}{ll}
{[b]_{i}^{(k)},} & i=1, \ldots, k, \\
{[b]_{i}^{(k)}-\frac{[a]_{i k}^{(k)}}{[a]_{k k}^{(k)}}[b]_{k}^{(k)},} & i=k+1, \ldots, n
\end{array}\right\}} \\
& {[x]_{i}^{G}=\left([b]_{i}^{(n)}-\sum_{j=i+1}^{n}[a]_{i j}^{(n)}[x]_{j}^{G}\right) /[a]_{i i}^{(n)}, \quad i=n, n-1, \ldots, 1 .}
\end{aligned}
$$

For theoretical considerations the multiplicative representation

$$
[x]^{G}=[D]^{(1)}\left([ U ] ^ { ( 1 ) } \left([ D ] ^ { ( 2 ) } \left([ U ] ^ { ( 2 ) } \left(\ldots \left([ U ] ^ { ( n - 1 ) } \left([ D ] ^ { ( n ) } \left([L]^{(n-1)}\left(\ldots\left([L]^{(2)}\left([L]^{(1)}[b]\right)\right) \ldots\right)\right.\right.\right.\right.\right.\right.\right.
$$

was presented in [55], where

$$
\begin{aligned}
& {[D]^{(k)}=I-e^{(k)}\left(e^{(k)}\right)^{\mathrm{T}}\left(1-\frac{1}{[a]_{k k}^{(k)}}\right),} \\
& {[L]^{(k)}=I-\left(e^{(k)}\left(0, \ldots, 0,[a]_{k+1, k}^{(k)}, \ldots,[a]_{n k}^{(k)}\right) /[a]_{k k}^{(k)}\right)^{\mathrm{T}},} \\
& {[U]^{(k)}=I-e^{(k)}\left(0, \ldots, 0,[a]_{k, k+1}^{(k)}, \ldots,[a]_{k n}^{(k)}\right) .}
\end{aligned}
$$

In [32] Neumaier obtained $[x]^{G}$ recursively via the partition

$$
[A]=\left(\begin{array}{cc}
{[a]_{11}} & {[c]^{\mathrm{T}}} \\
{[d]} & {[A]^{\prime}}
\end{array}\right), \quad[c],[d] \in \mathbb{I R}^{n-1},
$$

and the Schur complement $\Sigma_{[A]}^{G}=[A]^{\prime}-[d][c]^{\mathrm{T}} /[a]_{11} \in \mathbb{I}^{(n-1) \times(n-1)}$, provided that $0 \notin[a]_{11}$. He defines the triangular decomposition $([\hat{L}],[\hat{U}])$ of $[A]$ to exist, 
if either $n=1,[\hat{L}]=1,[\hat{U}]=[A] \not \supset 0$ or

$$
[\hat{L}]=\left(\begin{array}{cc}
1 & 0 \\
{[d] /[a]_{11}} & {[\hat{L}]^{\prime}}
\end{array}\right), \quad[\hat{U}]=\left(\begin{array}{cc}
{[a]_{11}} & {[c]^{\mathrm{T}}} \\
0 & {[\hat{U}]^{\prime}}
\end{array}\right)
$$

where $0 \notin[a]_{11}$ and where $\left([\hat{L}]^{\prime},[\hat{U}]^{\prime}\right)$ is the triangular decomposition of $\Sigma_{[A]}^{G}$. With the decomposition $[b]=\left([\beta],\left([b]^{\prime}\right)^{\mathrm{T}}\right)^{\mathrm{T}},[\beta] \in \mathbb{R},[b]^{\prime} \in \mathbb{I R}^{n-1}$ he finally ends up with the recursion

$$
[x]^{G}=\operatorname{IGA}([A],[b])=\left(\begin{array}{c}
{[x]_{1}^{G}} \\
\left([x]^{\prime}\right)^{G}
\end{array}\right),
$$

where $\left([x]^{\prime}\right)^{G}=\operatorname{IGA}\left(\Sigma_{[A]}^{G},[b]^{\prime}-[\beta][d] /[a]_{11}\right),[x]_{1}^{G}=\left([\beta]-[c]^{\mathrm{T}}\left([x]^{\prime}\right)^{G}\right) /[a]_{11}$.

If the triangular decomposition $([\hat{L}],[\hat{U}])$ exists then $[x]^{G}$ can equivalently be expressed by $[x]^{G}=\operatorname{IGA}([\hat{U}], \operatorname{IGA}([\hat{L}],[b]))$.

It is well-known that the feasibility of the conventional Gaussian algorithm is guaranteed if and only if all leading principal submatrices of $A$ are non-singular. Unfortunately, a similar criterion is missing for the interval version of the algorithm. Assuming that the interval Gaussian algorithm is feasible if and only if it is for every pair $(A, b) \in[A] \times[b]$ was shown to be false by Reichmann's counterexample [40]

$$
\left.[A]=\left(\begin{array}{ccc}
1 & {[0,2 / 3]} & {[0,2 / 3]} \\
{[0,2 / 3]} & 1 & {[0,2 / 3]} \\
{[0,2 / 3]} & {[0,2 / 3]} & 1
\end{array}\right) \quad \text { (modified as in }[31]\right) .
$$

Up to now only necessary or sufficient global criteria are known for the existence of $[x]^{G}$ unless $[A]$ is specialized. The most famous sufficient one is due to Alefeld [1]:

Theorem 5.1. Let $[A] \in \mathbb{I}^{n \times n}$ be an $H$-matrix and let $[b] \in \mathbb{I}^{n}$. Then $[x]^{G}$ exists.

This theorem applies at once to $M$-matrices, to generalized strictly dominant matrices, to generalized irreducibly diagonally dominant matrices and to generalized diagonally dominant matrices with $\langle[A]\rangle$ being non-singular. All of these are $H$-matrices.

In order to formulate a first class of matrices for which a necessary and sufficient criterion can be stated, we introduce the sign matrix $S$ of $[A]$ by $s_{i j}=\operatorname{sign}\left(\check{a}_{i j}\right)$ and the extended sign matrix $S^{\prime}$ by the following algorithm.

$$
S^{\prime}:=S
$$

for $k:=1$ to $n-1$ do

for $i:=k+1$ to $n$ do

for $j:=k+1$ to $n$ do

$$
\text { if } s_{i j}^{\prime}=0 \text { then } s_{i j}^{\prime}:=-s_{i k}^{\prime} s_{k k}^{\prime} s_{k j}^{\prime}
$$


Theorem $5.2([28])$. Let $[A] \in \mathbb{R}^{n \times n}, n>1$, be irreducible and generalized diagonally dominant and let $[b] \in \mathbb{R}^{n}$. Moreover, let $S^{\prime}$ be the extended sign matrix. Then $[x]^{G}$ exists if and only if $[A]$ is generalized irreducibly diagonally dominant or if

$$
s_{i j}^{\prime} s_{i k}^{\prime} s_{k k}^{\prime} s_{k j}^{\prime}= \begin{cases}1, & \text { if } i \neq j, \\ -1, & \text { if } i=j\end{cases}
$$

holds for some triple $(i, j, k)$ with $k<i, j$.

From Theorem 5.2 one deduces immediately that $[x]^{G}$ also exists if (5.1) holds with $S$ instead of $S^{\prime}$; cf. [14].

The subsequent result assumes $[A]$ to have the same form as in Theorem 4.1.

Theorem 5.3 ([29]). If $[A]=I+[-R, R] \in \mathbb{I}^{n \times n}, R \geq O$, then $[x]^{G}$ exists if and only if $\rho(R)<1$. This in turn holds if and only if $[A]$ is an $H$-matrix.

In order to formulate our third if-and-only-if-statement we use the concept of an undirected graph of a real matrix $A \in \mathbb{R}^{n \times n}$ with the nodes $1, \ldots, n$ and the edges $\{i, j\}$ if $a_{i j} \neq 0$ or $a_{j i} \neq 0$. We call $j$ a neighbor of the node $i(\neq j)$ if $i$ and $j$ are connected by an edge $\{i, j\}$. The number of neighbors of $i$ are the degree of $i$ in the underlying graph. Let $G_{k}$ denote the $k$-th elimination graph of $[A]$, i.e., the undirected graph of $\left|[A]^{(k)}\right|$ in which the nodes $1, \ldots, k-1$ and the corresponding edges have been removed and for which we assume that $[a]_{i j}^{(k-1)} \neq 0$ implies $[a]_{i j}^{(k)} \neq 0, i, j \geq k$ (no accidental zeros!). If in $G_{k}$ the node $k$ has the smallest degree and if this holds for all $k=1, \ldots, n$ then we say that $[A]$ is ordered by minimum degree.

Theorem 5.4 ([13]). Let $[A] \in \mathbb{I R}^{n \times n},[b] \in \mathbb{I R}^{n}$. If the (undirected) graph of $|[A]|$ is a tree (i.e., there are no cycles of length $\geq 3$ ) and if $[A]$ is ordered by minimum degree then $[x]^{G}$ exists if and only if $x^{G}$ exists for all $A \in[A]$.

Theorem 5.4 generalizes results of Reichmann [40] for tridiagonal matrices, of Garloff [15] for tridiagonal matrices with regular and totally non-negative element matrices, and of Schäfer [52] for arrowhead matrices. As an illustration of it and also of Theorem 5.2 we define the matrix $[A]$ as a circulant with the entries $[a]_{11}=$ $n(n-1) / 2,[a]_{1 j}=[0, j-1], j=2, \ldots, n$, in its first row and we let $[b]=[A] e$. The matrix $[A]$ is an unsymmetric Toeplitz matrix with $\langle[A]\rangle e=0$. Even when choosing $n=100$ and when taking into account rounding errors the maximal diameter of the components of $[x]^{G}$ remains less than 8 .

Now we address interval Hessenberg matrices.

Theorem 5.5 ([27], [39]). Let $[b] \in \mathbb{I}^{n}, n \geq 2$, and let $[A] \in \mathbb{I}^{n \times n}$ be an upper Hessenberg matrix satisfying $0 \notin[a]_{i i}, i=1, \ldots, n$, and $[a]_{i+1, i} \neq 0, i=$ $1, \ldots, n-1$. For $[a] \in \mathbb{I} \backslash\{\{0\}$ define $\sigma([a])=\operatorname{sign}(\check{a})$ if $0 \notin[a]$ and $\sigma([a])=0$ otherwise. Then $[x]^{G}$ exists if for each $i=1, \ldots, n-1$ and each $j=i+1, \ldots, n$ one of the following two conditions holds. 
(i) $[a]_{i j}=0 \Longrightarrow[a]_{p j}=0, p=1, \ldots, i-1$;

(ii) $[a]_{i j} \neq 0 \Longrightarrow \sigma\left([a]_{i i}\right) \sigma\left([a]_{i+1, j}\right)=-\sigma\left([a]_{i+1, i}\right) \sigma\left([a]_{i j}\right)$.

We close this section by mentioning that block variants of the interval Gaussian algorithm can be found in [17], pivoting is considered in [19], [45] and [61], criteria of infeasibility are presented in [25], perturbation results are stated in [32]. Division by zero is studied in [30]. For surveys on the algorithm see [13], [23], [27].

\section{Interval Cholesky method}

In order to enclose the symmetric solution set $\Sigma^{\text {sym }}$ the interval Cholesky method was introduced in [4]. It is defined analogously to the Cholesky method for point matrices and uses the square and square root function of interval arithmetic.

The algorithm starts with $[A]=[A]^{\mathrm{T}} \in \mathbb{R}^{n \times n},[b] \in \mathbb{R}^{n}$ and constructs the lower triangular matrix $[L]$ and the vectors $[y],[x]^{C}=\left([x]_{i}^{C}\right)=\operatorname{ICh}([A],[b]) \in \mathbb{I R}^{n}$ by

$$
\begin{aligned}
& {[l]_{j j}=\left([a]_{j j}-\sum_{k=1}^{j-1}[l]_{j k}^{2}\right)^{1 / 2}} \\
& \left.[l]_{i j}=\left([a]_{i j}-\sum_{k=1}^{j-1}[l]_{i k}[l]_{j k}\right) /[l]_{j j}, \quad i=j+1, \ldots, n\right\} j=1, \ldots, n ; \\
& {[y]_{i}=\left([b]_{i}-\sum_{j=1}^{i-1}[l]_{i j}[y]_{j}\right) /[l]_{i i}, \quad i=1, \ldots, n ;} \\
& {[x]_{i}^{C}=\left([y]_{i}-\sum_{j=i+1}^{n}[l]_{j i}[x]_{j}^{C}\right) /[l]_{i i}, \quad i=n, n-1, \ldots, 1 .}
\end{aligned}
$$

Apparently $[x]^{C}$ exists if and only if $0<\underline{l}_{i i}, i=1, \ldots, n$.

As for $[x]^{G}$ there is a representation of $[x]^{C}$ as a multiple product and in a recursive way. For the first one define the diagonal matrices $\left[D^{s}\right], s=1, \ldots, n$, and the lower triangular matrices $\left[L^{s}\right], s=1, \ldots, n-1$, by

$$
\left[d^{s}\right]_{i j}=\left\{\begin{array}{ll}
1 & \text { if } i=j \neq s, \\
1 /[l]_{s s} & \text { if } i=j=s, \\
0 & \text { otherwise }
\end{array} \quad\left[l^{s}\right]_{i j}= \begin{cases}1 & \text { if } i=j, \\
-[l]_{i s} & \text { if } i>j=s \\
0 & \text { otherwise }\end{cases}\right.
$$

Then

$$
\begin{aligned}
& {[y]=\left[D^{n}\right]\left(\left[L^{n-1}\right]\left(\left[D^{n-1}\right]\left(\ldots\left(\left[L^{2}\right]\left([D]^{2}\left(\left[L^{1}\right]\left(\left[D^{1}\right][b]\right)\right)\right)\right) \ldots\right)\right)\right)} \\
& {[x]^{C}=\left[D^{1}\right]\left(\left[L^{1}\right]^{\mathrm{T}}\left(\left[D^{2}\right]\left(\ldots\left(\left[L^{n-2}\right]^{\mathrm{T}}\left(\left[D^{n-1}\right]\left(\left[L^{n-1}\right]^{\mathrm{T}}\left(\left[D^{n}\right][y]\right)\right)\right)\right) \ldots\right)\right)\right) .}
\end{aligned}
$$

For the recursion we write the matrix $[A]=[A]^{\mathrm{T}} \in \mathbb{I}^{n \times n}$ as

$$
[A]=\left(\begin{array}{cc}
{[a]_{11}} & {[c]^{\mathrm{T}}} \\
{[c]} & {[A]^{\prime}}
\end{array}\right), \quad[c] \in \mathbb{R}^{n-1},
$$


and use its Schur complement $\Sigma_{[A]}^{C}=[A]^{\prime}-[c][c]^{\mathrm{T}} /[a]_{11}$ if $n>1,0 \notin[a]_{11}$, where this time $[c]_{i}[c]_{i}$ is evaluated as $[c]_{i}^{2}$. The Cholesky decomposition $\left([L],[L]^{\mathrm{T}}\right)$ of $[A]=[A]^{\mathrm{T}} \in \mathbb{I R}^{n \times n}$ is defined to exist if either $n=1,[L]=\left(\sqrt{[a]_{11}}\right)$ or if $n>1$ and

$$
[L]=\left(\begin{array}{cc}
\sqrt{\left[a_{11}\right]} & 0 \\
{[c] / \sqrt{\left[a_{11}\right]}} & {[L]^{\prime}}
\end{array}\right),
$$

where $0 \notin[a]_{11}$ and where $\left([L]^{\prime},\left([L]^{\prime}\right)^{\mathrm{T}}\right)$ is the Cholesky decomposition of $\Sigma_{[A]}^{C}$.

It is shown in [4] that the matrix $[L]$ for the Cholesky decomposition is the same as for the interval Cholesky method.

The output vector $[x]^{C}$ generally differs from $[x]^{G}$ and can satisfy $[x]^{C} \subseteq[x]^{G}$ or $[x]^{C} \supseteq[x]^{G}$ or none of these relations. As an example choose $[A]=\left(\begin{array}{c}4 \\ {[-1,1]}\end{array}{ }^{[-1,1]}\right)$ and $[b]=(6,6)^{\mathrm{T}}$. Then $\Sigma=$ convex hull $\left(\left(\frac{6}{5}, \frac{6}{5}\right)^{\mathrm{T}},(2,2)^{\mathrm{T}},\left(\frac{18}{17}, \frac{30}{17}\right)^{\mathrm{T}},\left(\frac{30}{17}, \frac{18}{17}\right)^{\mathrm{T}}\right) \supseteq$ $\Sigma^{\mathrm{sym}}=\left\{\gamma(1,1)^{\mathrm{T}} \mid \frac{6}{5} \leq \gamma \leq 2\right\}$ and $[x]^{G}=\left([1,2],\left[\frac{18}{17}, 2\right]\right)^{\mathrm{T}} \supseteq[x]^{C}=\left([1,2],\left[\frac{18}{16}, 2\right]\right)^{\mathrm{T}}$.

It is well-known that the Cholesky decomposition exists for $A=A^{\mathrm{T}} \in \mathbb{R}^{n \times n}$ if and only if $A$ is symmetric and positive definite. Reichmann's example in [40] shows however again that this criterion has no interval analogue. Therefore, one has to proceed as in Section 5. In [7] we proved the following result.

TheOREM 6.1. Let $[b] \in \mathbb{R}^{n}$ and let $[A]=[A]^{\mathrm{T}} \in \mathbb{I}^{n \times n}$ contain a symmetric and positive definite matrix $\tilde{A}$. If $[x]^{G}$ exists then $[x]^{C}$ exists, too.

For $n \leq 3$ this result is reversible, but unfortunately not for $n \geq 4$ as was shown by an example in [7]. One only can prove the following weaker result.

TheOREM $6.2([7])$. Let $[b] \in \mathbb{R}^{n}$ and let all symmetric matrices $\tilde{A} \in[A]=$ $[A]^{\mathrm{T}} \in \mathbb{I R}^{n \times n}$ be positive definite. Then the Gaussian algorithm is feasible for all matrices $A \in[A]$.

Based on Theorem 6.1 one obtains the analogue of Theorem 5.1.

Theorem 6.3 ([4]). Let $[A]=[A]^{\mathrm{T}} \in \mathbb{I R}^{n \times n}$ be an $H$-matrix with $\underline{a}_{i i}>0$ for $i=1, \ldots, n$ and let $[b] \in \mathbb{R}^{n}$. Then $[x]^{C}$ exists.

If one requires $[A]=[A]^{\mathrm{T}}$ to satisfy $\underline{a}_{i i}>0$ then one can replace $[x]^{G}$ by $[x]^{C}$ in Theorems 5.2 and 5.3; cf. [7] for details. The criterion in Theorem 5.2 can be shortened slightly, leading to our next result.

TheOREM 6.4 ([7]). Let $[b] \in \mathbb{I}^{n}$ and let $[A]=[A]^{\mathrm{T}} \in \mathbb{R}^{n \times n}$ be irreducible and generalized diagonally dominant with $0<\underline{a}_{i i}, i=1, \ldots, n$. Moreover, let $S^{\prime}$ be the extended sign matrix of $[A]$ defined in Section 5 . Then $[x]^{C}$ exists if and only if $[A]$ is generalized irreducibly diagonally dominant or the sign condition $s_{i j}^{\prime} s_{i k}^{\prime} s_{k j}^{\prime}=1$ holds for some triple $(i, j, k)$ with $k<j<i$.

Theorem 5.4 can also be adapted. 
TheOrem 6.5 ([7]). Let $[b] \in \mathbb{I}^{n}$ and let $[A]=[A]^{\mathrm{T}} \in \mathbb{I}^{n \times n}$ contain a symmetric and positive definite matrix. If the (undirected) graph of $|[A]|$ is a tree and if $[A]$ is ordered by minimum degree then $[x]^{C}$ exists if and only if $[x]^{G}$ exists.

We also mention Frommer's paper [13] in which a variant of the interval Cholesky method is presented, and Schäfer's paper [53] in which block variants for the method are considered. Perturbation results are contained in [5].

\section{Interval Toeplitz methods}

We start this section with real $(n+1) \times(n+1)$ Toeplitz matrices

$$
A=\left(\begin{array}{cccc}
\tau_{0} & \tau_{1} & \cdots & \tau_{n} \\
\tau_{-1} & \tau_{0} & \ddots & \vdots \\
\vdots & \ddots & \ddots & \tau_{1} \\
\tau_{-n} & \cdots & \tau_{-1} & \tau_{0}
\end{array}\right)
$$

where $a_{i j}=\tau_{j-i}$ and where we assume that all leading principal submatrices $A_{k} \in$ $\mathbb{R}^{k \times k}$ of $A$ are regular so that the Gaussian algorithm is feasible. (For ease of presentation we consider $(n+1) \times(n+1)$ matrices instead of $n \times n$ ones.) One sees directly that $A$ is persymmetric, and that its inverse is persymmetric, too. Since $A$ is a Toeplitz matrix if and only if $E A$ is a Hankel matrix, the subsequent results can be transferred to Hankel matrices without any problems. A Toeplitz matrix (7.1) depends only on $2 n+1$ parameters $\tau_{i}$. Therefore, it can be expected that there are direct methods which solve a Toeplitz system with far less than $O\left(n^{3}\right)$ operations. In fact, for the following algorithm of Trench $O\left(n^{2}\right)$ operations are sufficient. The algorithm as presented here is essentially based on Zohar's work [62]. See also [60] and [34]. There are two major steps.

In the first step the vectors $l_{k}, u_{k} \in \mathbb{R}^{k}, k=1, \ldots, n$ and the corresponding real numbers $\delta_{k}$ are computed recursively for the representation

$$
A_{k+1}=\left(\begin{array}{cc}
A_{k} & \overleftarrow{t_{k}} \\
\overleftarrow{t}_{-k}^{\mathrm{T}} & \tau_{0}
\end{array}\right)=\left(\begin{array}{cc}
I_{k} & 0 \\
-l_{k}^{\mathrm{T}} & 1
\end{array}\right)\left(\begin{array}{cc}
A_{k} & 0 \\
0 & \delta_{k}
\end{array}\right)\left(\begin{array}{cc}
I_{k} & -u_{k} \\
0 & 1
\end{array}\right)
$$

of the leading principal submatrices of $A$, where $t_{k}=\left(\tau_{1}, \ldots, \tau_{k}\right)^{\mathrm{T}}, t_{-k}=$ $\left(\tau_{-1}, \ldots, \tau_{-k}\right)^{\mathrm{T}} \in \mathbb{R}^{k}$ and where $I_{k}$ is the $k \times k$ unit matrix.

In the second step the entry $\delta_{k}$ in (7.2) is used to construct recursively the vectors $x^{(k)}, k=1, \ldots, n+1$, which satisfy

$$
A_{k} x^{(k)}=b^{(k)}=\left(b_{1}, \ldots, b_{k}\right)^{\mathrm{T}} .
$$

Then the final vector $x^{(n+1)}$ is the solution of the initial Toeplitz system $A x=b$. 
For the first step and $k=0, \ldots, n-1$ one computes

$$
\left\{\begin{array}{l}
\delta_{k}=\left(\operatorname{det} A_{k+1}\right) /\left(\operatorname{det} A_{k}\right)=\tau_{0}+\overleftarrow{t}_{-k}^{\mathrm{T}} u_{k}(\text { also for } k=n) \\
\gamma_{k}=-\left(t_{k}^{\mathrm{T}} u_{k}+\tau_{k+1}\right) / \delta_{k}, \quad \hat{\gamma}_{k}=-\left(\overleftarrow{t}_{-k}^{\mathrm{T}} \overleftarrow{l}_{k}+\tau_{-k-1}\right) / \delta_{k} \\
u_{k+1}=\left(\begin{array}{c}
0 \\
u_{k}
\end{array}\right)+\gamma_{k}\left(\begin{array}{c}
1 \\
\overleftarrow{l}_{k}
\end{array}\right), \quad \overleftarrow{l}_{k+1}=\left(\begin{array}{c}
\overleftarrow{l}_{k} \\
0
\end{array}\right)+\hat{\gamma}_{k}\left(\begin{array}{c}
u_{k} \\
1
\end{array}\right)
\end{array}\right.
$$

where in the case $k=0$ we assume that the terms $A_{0}, u_{0}, \overleftarrow{l}_{0}, t_{0} \overleftarrow{t}_{-0}^{\mathrm{T}}$ are not present

For the second step one starts with

$$
A_{k+1}\left\{x^{(k+1)}-\left(\begin{array}{c}
x^{(k)} \\
0
\end{array}\right)\right\}=\left(\begin{array}{c}
0 \\
b_{k+1}-\overleftarrow{t}_{-k}^{\mathrm{T}} x^{(k)}
\end{array}\right)
$$

which implies the recursion

$$
\left.\begin{array}{l}
\zeta_{k}=\left(b_{k+1}-\overleftarrow{t}_{-k}^{\mathrm{T}} x^{(k)}\right) / \delta_{k}, \\
x^{(k+1)}=\left(\begin{array}{c}
x^{(k)} \\
0
\end{array}\right)+\zeta_{k}\left(\begin{array}{c}
u_{k} \\
1
\end{array}\right)
\end{array}\right\} k=0, \ldots, n .
$$

Here again the terms $u_{0}, \overleftarrow{t}_{-0}^{\mathrm{T}}, x^{(0)}$ are assumed not to be present if $k=0$

Introducing interval brackets in (7.4), (7.5), one obtains the interval Trench algorithm, which is a modification of Garloff's algorithm ITA in [16]. In contrast to there, we do not normalize $[A]$ in the diagonal.

The enclosure $\Sigma^{\text {Toep }} \subseteq[x]^{\text {ITA }}$ follows immediately, but again Reichmann's counterexample from Section 5 applies. The following theoretical results are partly known for the unmodified variant in [16].

TheOREM 7.1. If $[A]$ is a Toeplitz $H$-matrix, then $[x]^{\mathrm{ITA}}$ exists.

TheOrem 7.2. Let $[A]=I+[-R, R]$ be a Toeplitz matrix with $R \geq O$. Then $[x]^{\mathrm{ITA}}$ exists if and only if $\rho(R)<1$ hence if and only if $[A]$ is an $H$-matrix.

TheOREm 7.3. Let $[A]=[A]^{\mathrm{T}}$ be tridiagonal and let there exist a symmetric positive definite matrix $\tilde{A} \in[A]$ with $\langle\tilde{A}\rangle=\langle[A]\rangle$. Then $[x]^{\mathrm{ITA}}$ exists.

As a second algorithm for real $(n+1) \times(n+1)$ Toeplitz systems, we recall Bareiss' algorithm [8]. Starting with $A^{(0)}=A$, it constructs recursively a sequence of matrices $A^{(-k)}, A^{(k)}, k=0,1, \ldots, n$, in which the first $k$ subdiagonals below the main diagonal and above, respectively, are eliminated. For $k>0$ the matrices $A^{(-k)}, A^{(k)}$ have the block form

$$
A^{(-k)}=\left(\begin{array}{c}
U^{(-k)} \\
T^{(-k)}
\end{array}\right), \quad A^{(k)}=\left(\begin{array}{c}
T^{(k)} \\
L^{(k)}
\end{array}\right),
$$

where $U^{(-k)} \in \mathbb{R}^{(k+1) \times(n+1)}$ consists of the first $k+1$ rows of an upper triangular matrix, $L^{(k)} \in \mathbb{R}^{(k+1) \times(n+1)}$ consists of the last $k+1$ rows of a lower triangular 
matrix, and $T^{(-k)}, T^{(k)} \in \mathbb{R}^{(n-k) \times(n+1)}$ are rectangular Toeplitz matrices with zeros in position $2, \ldots, k+1$ of its first row. For the elimination process, choose $\alpha_{-k}$ such that the Toeplitz matrix $\bar{T}^{(-k)}=T^{(-k)}-\alpha_{-k} T^{(k)}$ has an additional zero in position 1 of its first row and define $A^{(-k-1)}=\left(\frac{U^{(-k)}}{T^{(-k)}}\right)$. Then adapt $\alpha_{k}$ such that the Toeplitz matrix $\bar{T}^{(k)}=T^{(k)}-\alpha_{k} \bar{T}^{(-k)}$ has an additional zero in position $k+2$ of its first row and hence in position $n+1$ of its last row, and set $A^{(k+1)}=\left(\begin{array}{l}\bar{T}^{(k)} \\ L^{(k)}\end{array}\right)$. Now append the first row of $\bar{T}^{(-k)}$ to $U^{(-k)}$ from below to obtain $U^{(-k-1)}$ leaving $T^{-k-1}$, and similarly the last row of $\bar{T}^{(k)}$ to the top of $L^{(k)}$ to obtain $L^{(k+1)}$ leaving $U^{(k+1)}$. Transforming the righthand side $b$ in the same way and replacing $k$ by $k+1$ finally results in the following recursion

$$
b^{(0)}=b, \quad \tau_{j}^{(0)}=\tau_{j}, j=0, \pm 1, \ldots, \pm n,
$$

and for $k=1,2, \ldots, n$ :

$$
\begin{array}{ll}
\tau_{j}^{(-k)}=\tau_{j}^{(-k+1)}-\frac{\tau_{-k}^{(-k+1)}}{\tau_{0}} \tau_{j+k}^{(k-1)}, & \\
j=-n,-n+1, \ldots,-k-1 ; 0,1, \ldots, n-k & \text { for } k<n, \\
j=0 & \text { for } k=n, \\
\tau_{j}^{(k)}=\tau_{j}^{(k-1)}-\frac{\tau_{j}^{(k-1)}}{\tau_{0}^{(-k)}} \tau_{j-k}^{(-k)}, & \text { for } k<n, \\
j=-n+k,-n+k+1, \ldots,-1 ; k+1, \ldots, n & \\
b_{j}^{(-k)}=b_{j}^{(-k+1)}-\frac{\tau_{-k}^{(-k+1)}}{\tau_{0}} b_{j-k}^{(k-1)}, \quad j=k, k+1, \ldots, n, \\
b_{j}^{(k)}=b_{j}^{(k-1)}-\frac{\tau_{k}^{(k-1)}}{\tau_{0}^{(-k)}} b_{j+k}^{(-k)}, \quad j=1, \ldots, n+1-k .
\end{array}
$$

Here we exploited the fact that $\tau_{0}=\tau_{0}^{(1)}=\cdots=\tau_{0}^{(k)}=\cdots=\tau_{0}^{(n)}$.

At the end we get

$$
A^{(-n)}=\left(\begin{array}{cccc}
\tau_{0}^{(0)} & \tau_{1}^{(0)} & \cdots & \tau_{n}^{(0)} \\
0 & \tau_{0}^{(-1)} & \cdots & \tau_{n-1}^{(-1)} \\
\vdots & \ddots & \ddots & \vdots \\
0 & \cdots & 0 & \tau_{0}^{(-n)}
\end{array}\right) \text { and } A^{(n)}=\left(\begin{array}{cccc}
\tau_{0} & 0 & \cdots & 0 \\
\tau_{-1}^{(n-1)} & \tau_{0} & \cdots & 0 \\
\vdots & \ddots & \ddots & \vdots \\
\tau_{-n}^{(0)} & \cdots & \tau_{-1}^{(0)} & \tau_{0}
\end{array}\right)
$$

The solution of the original system can be obtained by the upper half of the system $A^{(n)} x=b^{(n)}$ and the lower half of the system $A^{(-n)} x=b^{(-n)}$ saving more than one half of operations as compared with a complete forward resp. backward substitution of one of these triangular systems. 
The corresponding interval method just plugs in brackets around the items in (7.6), (7.7) and will not be repeated here. It is an unsymmetric variant of Garloff's interval Bareiss method in [17] and results in an interval vector $[x]^{\text {IBA }} \supseteq \Sigma^{\text {Toep }}$. One can show that the Theorems 7.1-7.3 hold for this second interval method, too. Garloff reports in [17] that for most of his examples he got the inclusion $[x]^{\mathrm{IBA}} \subseteq[x]^{G} \subseteq[x]^{\mathrm{ITA}}$.

\section{Further enclosures for $\Sigma$}

Our final section is devoted to enclosures for $\Sigma$ which often form the starting point for iterative methods. These latter ones seem to be applied to interval linear systems more frequently than direct ones. Under some additional conditions they do not break down and deliver verification mostly in a few steps. These advantages may hide the fact that they require a stopping criterion when computing on a machine.

Our first result is based on Richardson's splitting for $[A]$ and on some arbitrary vector $\tilde{x}$ which is usually an approximation of the solution of some particular system of (1.1).

Theorem 8.1. Let $[A] \in \mathbb{R}^{n \times n},[C]=I-[A]$, and $[b] \in \mathbb{I}^{n \times n}$. Moreover, choose $\tilde{x} \in \mathbb{R}^{n}$.

a) If

$$
([C][x]+[b])_{i} \varsubsetneqq[x]_{i}, \quad i=1, \ldots, n
$$

for some vector $[x] \in \mathbb{R}^{n}$ then $\rho(|[C]|)<1$ and $\Sigma \subseteq[x]$.

b) If $\||[C]|\|_{\infty}<1$ then $\Sigma \subseteq \tilde{x}+\frac{\|[b]-[A] \tilde{x} \mid\|_{\infty}}{1-\|\left[|[C]| \|_{\infty}\right.}[-e, e]$, where $\|\cdot\|_{\infty}$ denotes the usual maximum norm and the row sum norm, respectively.

Condition (8.1) is the key for verification in practice as was demonstrated intensively by Rump; cf. [48]. It is mostly applied to (1.2) after preconditioning with some real matrix $R$. In this way one obtains the following result.

Theorem $8.2([20],[49])$. Let $[A] \in \mathbb{R}^{n \times n}, R \in \mathbb{R}^{n \times n},[b],[x] \in \mathbb{I R}^{n}, \tilde{x} \in$ $[x]$. Moreover define $[d]=R([b]-[A] \tilde{x}),[C]=I-R[A],[x]_{\Delta}=[x]-\tilde{x},[y]_{\Delta}=$ $[d]+[C][x]_{\Delta}$. If $\left([y]_{\Delta}\right)_{i} \varsubsetneqq\left([x]_{\Delta}\right)_{i}, i=1, \ldots, n$, then the following properties hold.

a) The matrices $R$ and $[A]$ are regular, $\rho(|[C]|)<1, \Sigma \subseteq \tilde{x}+[x]_{\Delta}$.

b) $\min \left(\Sigma_{i}\right) \in \tilde{x}_{i}+\underline{d}_{i}+\left([C][x]_{\Delta}\right)_{i}, i=1, \ldots, n, \max \left(\Sigma_{i}\right) \in \tilde{x}_{i}+\bar{d}_{i}+\left([C][x]_{\Delta}\right)_{i}, i=$ $1, \ldots, n$, where $\Sigma_{i}$ denotes the projection of $\Sigma$ onto the $i$-th coordinate axis.

c) If $[A]=[A]^{\mathrm{T}}$ and if $[d]_{i}, i=1, \ldots, n$, is replaced by

$$
[d]_{i}^{\mathrm{sym}}=\sum_{j=1}^{n} r_{i j}\left([b]_{j}-[a]_{j j} \tilde{x}_{j}\right)-\sum_{j=1}^{n} \sum_{l=1}^{j-1}\left(r_{i j} \tilde{x}_{l}+r_{i l} \tilde{x}_{j}\right)[a]_{j l}
$$

then b) holds with $\Sigma^{\mathrm{sym}}$ instead of $\Sigma$. 
Items b) and c) are starting points for a sensitivity analysis since they present error bounds for the overestimation of $\tilde{x}+[x]_{\Delta}$ over $\Sigma$ and $\Sigma^{\text {sym }}$, respectively.

Sometimes the condition (8.1) or its analogous condition in Theorem 8.2 are not fulfilled. Then $\varepsilon$-inflation may help; cf. [24], [48], [50] and the literature therein.

We finally recall that singular interval systems (1.3) are defined and studied in [6], [26], [42], [44], [46]. Cyclic reduction as a direct method for tridiagonal systems is introduced in [56]. Pagelimit did not allow us to present particular methods for enclosing the solution of a single linear system. As examples we only mention [37], [38]. We also could not consider the variety of very efficient iterative methods for enclosing $\Sigma$. Here, starting points might be [1], [32], [48], [51] and the literature therein.

\section{References}

[ 1 ] G. Alefeld and J. Herzberger, Introduction to Interval Computations. Academic Press, New York, 1983.

[ 2 ] G. Alefeld, V. Kreinovich and G. Mayer, On the shape of the symmetric, persymmetric and skew-symmetric solution set. SIAM J. Matrix Anal. Appl., 18 (1997), 693-705.

[ 3 ] G. Alefeld, V. Kreinovich and G. Mayer, On the solution sets of particular classes of linear interval systems. J. Comp. Appl. Math., 152 (2003), 1-15.

[ 4 ] G. Alefeld and G. Mayer, The Cholesky method for interval data. Linear Algebra Appl., 194 (1993), 161-182.

[5] G. Alefeld and G. Mayer, On the symmetric and unsymmetric solution set of interval systems. SIAM J. Matrix Anal. Appl., 16 (1995), 1223-1240.

[ 6 ] G. Alefeld and G. Mayer, Enclosing solutions of singular interval systems iteratively. Reliable Computing, 11 (2005), 165-190.

[ 7 ] G. Alefeld and G. Mayer, New criteria for the feasibility of the Cholesky method with interval data. SIAM J. Matrix Anal. Appl., 30 (2008), 1392-1405.

[8] E.H. Bareiss, Numerical solution of linear equations with Toeplitz and vector Toeplitz matrices. Numer. Math., 13 (1969), 404-424.

[ 9 ] W. Barth and E. Nuding, Optimale Lösung von Intervallgleichungssystemen, Computing, 12 (1974), 117-125.

[10] H. Beeck, Über Struktur und Abschätzungen der Lösungsmenge von linearen Gleichungssystemen mit Intervallkoeffizienten. Computing, 10 (1972), 231-244.

[11] H. Beeck, Zur scharfen Außenabschätzung der Lösungsmenge bei linearen Intervallgleichungssystemen. Z. Angew. Math. Mech., 54 (1974), T208-T209.

[12] C. Bliek, Computer methods for design automation. Ph.D. Thesis, Massachusetts Institute of Technology, Cambridge, MA, 1992.

[13] A. Frommer, A feasibility result for interval Gaussian elimination relying on graph structure. Symbolic Algebraic Methods and Verification Methods, G. Alefeld, J. Rohn, S. Rump and T. Yamamoto (eds.), Springer, Wien, 2001, 79-86.

[14] A. Frommer and G. Mayer, A new criterion to guarantee the feasibility of the interval Gaussian algorithm. SIAM J. Matrix Anal. Appl., 14 (1993), 408-419.

[15] J. Garloff, Totally nonnegative interval matrices. Interval Mathematics 1980, K.L.E. Nickel (ed.), Academic Press, New York, 1980, 317-327.

[16] J. Garloff, Solution of linear equations having a Toeplitz interval matrix as coefficient matrix. Opuscula Mathematica, 2 (1986), 33-45.

[17] J. Garloff, Block methods for the solution of linear interval equations. SIAM J. Matrix Anal. Appl., 11 (1990), 89-106.

[18] E.R. Hansen, Bounding the solution of interval linear equations. SIAM J. Numer. Anal., 29 (1992), 1493-1503.

[19] M. Hebgen, Eine scaling-invariante Pivotsuche für Intervallmatrizen. Computing, 12 (1974), 99-106. 
[20] C. Jansson, Interval linear systems with symmetric matrices, skew-symmetric matrices and dependencies in the right hand side. Computing, 46 (1991), 265-274.

[21] C. Jansson, Calculation of exact bounds for the solution set of linear interval systems. Linear Algebra Appl., 251 (1997), 321-340.

[22] V. Kreinovich, A. Lakeyev, J. Rohn and P. Kahl, Computational Complexity and Feasibility of Data Processing and Interval Computations. Kluwer, Dordrecht, 1998.

[23] G. Mayer, Old and new aspects of the interval Gaussian algorithm. Computer Arithmetic, Scientific Computation and Mathematical Modelling, E. Kaucher, S.M. Markov and G. Mayer (eds.), Baltzer, Basel, 1991, 329-349.

[24] G. Mayer, Epsilon-inflation with contractive interval functions. Appl. Math., 43 (1998), 241-254.

[25] G. Mayer, A contribution to the feasibility of the interval Gaussian algorithm. Reliable Computing, 12 (2006), 79-98.

[26] G. Mayer, On regular and singular interval systems, J. Comp. Appl. Math., 199 (2007), $220-228$

[27] G. Mayer, On the interval Gaussian algorithm. IEEE-Proceedings of SCAN 2006, IEEE Computer Society, Washington DC, 2007.

[28] G. Mayer and L. Pieper, A necessary and sufficient criterion to guarantee the feasibility of the interval Gaussian algorithm for a class of matrices. Appl. Math., 38 (1993), 205-220.

[29] G. Mayer and J. Rohn, On the applicability of the interval Gaussian algorithm. Reliable Computing, 4 (1998), 205-222.

[30] J. Mayer, An approach to overcome division by zero in the interval Gaussian algorithm. Reliable Computing, 8 (2002), 229-237.

[31] A. Neumaier, New techniques for the analysis of linear interval equations. Linear Algebra Appl., 58 (1984), 273-325.

[32] A. Neumaier, Interval Methods for Systems of Equations. Cambridge University Press, Cambridge, 1990

[33] A. Neumaier, A simple derivation of the Hansen-Bliek-Rohn-Ning-Kearfott enclosure for linear interval equations. Reliable Computing, 5 (1999), 131-136.

[34] M.K. Ng, Iterative Methods for Toeplitz Systems. Oxford Univ. Press, Oxford, 2004.

[35] S. Ning and R.B. Kearfott, A comparison of some methods for solving linear interval equations. SIAM J. Numer. Anal., 34 (1997), 1289-1305.

[36] W. Oettli and W. Prager, Compatibility of approximate solution of linear equations with given error bounds for coefficients and right-hand sides. Numer. Math., 6 (1964), 405-409.

[37] T. Ogita, S. Oishi and Y. Ushiro, Computation of sharp rigorous componentwise error bounds for the approximate solutions of systems of linear equations. Reliable Computing, 9 (2003), 229-239.

[38] S. Oishi and S.M. Rump, Fast verification of solutions of matrix equations. Numer. Math., 90 (2002), 755-773.

[39] K. Reichmann, Ein hinreichendes Kriterium für die Durchführbarkeit des Intervall-GaußAlgorithmus bei Intervall-Hessenberg-Matrizen ohne Pivotsuche. Z. Angew. Math. Mech., 59 (1979), 373-379.

[40] K. Reichmann, Abbruch beim Intervall-Gauss-Algorithmus. Computing, 22 (1979), 355-361.

[41] J. Rohn, Inner solutions of linear interval systems. Interval Mathematics 1985, K. Nickel (ed.), Lecture Notes in Computer Science, 212, Springer, Berlin, 1986, 157-158.

[42] J. Rohn, Systems of linear interval equations. Linear Algebra Appl., 126 (1989), 39-78.

[43] J. Rohn, Cheap and tight bounds: The recent result by E. Hansen can be made more efficient. Interval Computations, 4 (1993), 13-21.

[44] J. Rohn, Interval matrices: Singularity and real eigenvalues. SIAM J. Matrix Anal. Appl., 14 (1993), 82-91.

[45] J. Rohn, On overestimation produced by the interval Gaussian algorithm. Reliable Computing, 3 (1997), 363-368.

[46] J. Rohn, A Handbook of Results on Interval Linear Problems. April 7, 2005, http:// www.cs.cas.cz/ rohn/handbook/.

[47] J. Rohn and V. Kreinovich, Computing exact componentwise bounds on solutions of linear systems with interval data is NP-hard. SIAM J. Matrix Anal. Appl., 16 (1995), 415-420. 
[48] S.M. Rump, Solving algebraic problems with high accuracy. A New Approach to Scientific Computation, U.W. Kulisch and W.L. Miranker (eds.), Academic Press, New York, 1983, 51-120.

[49] S.M. Rump, Rigorous sensitivity analysis for systems of linear and nonlinear equations. Math. Comp., 54 (1990), 721-736.

[50] S.M. Rump, On the solution of interval linear systems. Computing, 47 (1992), 337-353.

[51] S.M. Rump, Verification methods for dense and sparse systems of equations. Topics in Validated Computations, J. Herzberger (ed.), Elsevier, Amsterdam, 1994, 63-135.

[52] U. Schäfer, The feasibility of the interval Gaussian algorithm for arrowhead matrices. Reliable Computing, 7 (2001), 59-62.

[53] U. Schäfer, Two ways to extend the Cholesky decomposition to block matrices with interval entries. Reliable Computing, 8 (2002), 1-20.

[54] F. Schätzle, Überschätzung beim Gauss-Algorithmus für lineare Intervallgleichungssysteme. Freiburger Intervall-Berichte, 84/3 (1984), 1-122.

[55] H. Schwandt, An interval arithmetic approach for the construction of an almost globally convergent method for the solution of nonlinear Poisson equation on the unit square. SIAM J. Sci. Statist. Comput., 5 (1984), 427-452.

[56] H. Schwandt, Cyclic reduction for tridiagonal systems of equations with interval coefficients on vector computers. SIAM J. Numer. Anal., 26 (1989), 661-680.

[57] S.P. Shary, Optimal solution of interval linear algebraic systems, I. Interval Computations, 2 (1991), 7-30.

[58] S.P. Shary, A new technique in systems analysis under interval uncertainty and ambiguity. Reliable Computing, 8 (2002), 321-418.

[59] G.W. Stewart, Matrix Algorithms, Vol. I: Basic Decompositions. SIAM, Philadelphia, 1998.

[60] W.F. Trench, An algorithm for the inversion of finite Toeplitz matrices. J. Soc. Indust. Appl. Math., 12 (1964), 515-522.

[61] P. Wongwises, Experimentelle Untersuchungen zur numerischen Auflösung von linearen Gleichungssystemen mit Fehlererfassung. Interval Mathematics, G. Goos and J. Hartmanis (eds.), Lecture Notes in Computer Science, 29, Springer, Berlin, 1975, 316-325.

[62] S. Zohar, The solution of a Toeplitz set of linear equations. J. Assoc. Comput. Mach., 21 (1974), 272-276. 